\title{
整系数多项式模 $2^{d}$ 本原性的判别
}

\author{
戴 宗 锋 \\ 黄 民强 \\ (中国科学院研究生院, 北京 100039) \\ (中国科学院系统科学研究所, 北京 100080)
}

关锽词多项式、模 $2^{a}$ 本原性、判别式

Galois 域 $F_{2}$ 上多项式的本原性已经有充分的研究 ${ }^{[1,21}$. 将这个问题推广到 整 数 剩 余 类环 $\boldsymbol{Z} /\left(2^{d}\right)$ 上 $(d \geqslant 2)$ 具有理论和实际意义. 类似于模 2 的情形, 对于 $f(x)-x^{n}+$ $c_{*-1} x^{*-1}+\cdots+c_{0} \in \boldsymbol{Z}[x]$, 其中 $\left(c_{0}, 2\right)-1$, 我们可以定义其模 $2^{d}$ 的周期(记作 $\left.\operatorname{per}(f)_{2}^{d}\right)$ 为满足下式的最小正整数 $t$ :

$$
x^{t} \equiv 1, \bmod \left(2^{d}, f(x)\right) .
$$

容易证明 ${ }^{[3,4]} \operatorname{per}(f)_{2^{d}}-2^{e} \operatorname{per}(f)_{2}, e \leqslant d-1$. 于是有周期的上界 $\operatorname{per}(f)_{2^{d}} \leqslant 2^{d-1}\left(2^{n}-1\right)$. 同 $\boldsymbol{F}_{2}$ 上一样, 我们称 $f(x)$ 在 $\boldsymbol{Z} /\left(2^{d}\right)$ 上本原, 如果 $\operatorname{per}(f)_{2^{d}}-2^{d-1}\left(2^{-}-1\right)$. 此时 $f(x)$ 显然在 $\boldsymbol{F}_{2}$ 上本原, 以下总假设如此. 本文将给出一个 $\boldsymbol{Z} /\left(2^{d}\right)$ 上本原性的判别式. 而

设 $f(x)$ 有二进制表达式 $f(x)-\sum_{i=0}^{a-1} 2^{i} f_{i}(x), f_{i}(x) \in F_{2}[x]$, 并且 $i \geqslant 1$ 时 $\operatorname{deg} f_{i}(x)<n$,

$$
f_{0}(x)-\sum_{i \in S} x^{i}
$$

由于 $\operatorname{deg} f_{0}(x)=n, f_{0}(x)$ 在 $\boldsymbol{F}_{2}$ 上本原, 我们有 $T=\operatorname{per}\left(f_{0}\right)=\operatorname{per}(f)_{2}-2^{*}-1$. 在 $\boldsymbol{F}_{2}[x]$ 中取 $k(x)=\left(x^{T}+1\right) / f_{0}(x)$, 则在 $\boldsymbol{F}_{2}$ 上有

$$
x^{T}=1+k(x) f_{0}(x) .
$$

在 $\boldsymbol{Z}[\boldsymbol{x}]$ 中考虑上式,有

$$
\begin{aligned}
x^{T} & =1+k(x) f_{0}(x)+2 h(x) \\
& \equiv 1+2\left(h(x)-k(x) \sum_{i>1} 2^{i-1} f_{i}(x)\right),(\bmod f(x)),
\end{aligned}
$$

其中 $h(x) \in \boldsymbol{Z}[x]$ 由 $f_{0}(x)$ 唯一确定. 令

则对 $i \geqslant 1$ 有

$$
\begin{aligned}
& h_{1}(x)=h(x)-k(x) \sum_{i>1} 2^{i-1} f_{i}(x), \\
& h_{i+1}(x)=h_{i}(x)+2^{i-1} h_{i}^{i}(x),
\end{aligned}
$$

$$
x^{2^{2-i} T} \equiv 1+2^{i} h_{i}(x),(\bmod f(x)) .
$$

由上式可见 $\operatorname{per}(f)_{i^{i}} \mid 2^{i-1} T$.

本文 1990 年 1 月 3 日收到. 
引理 1 对 $i \geqslant 1, \operatorname{per}(f)_{2^{i+1}}=2^{i} T$ 当且仅当

$$
h_{i}(x) \neq 0, \bmod (2, f(x)) \text {. }
$$

证 必要性显然. 充分性可由下述易证的引理推得.

引理 2 设 $k \geqslant 1$, 任意 $g(x) \in \boldsymbol{Z}[x]$ 可唯一写成

$$
g(x)=g_{1}(x)+2^{k} g_{2}(x)+f(x) g_{3}(x),
$$

其中 $\operatorname{deg} g_{1}(x), \operatorname{deg} g_{2}(x)<\operatorname{deg} f(x)$, 且 $g_{1}(x)$ 的系数介于 0 与 $2^{k}-1$ 之间. 更进一步 地, $g(\boldsymbol{x}) \in\left(2^{k}, f(\boldsymbol{x})\right)$ 当且仅当 $g_{1}(x)=0$.

设 $\pi$ 是 $f_{0}(x)$ (或 $\left.f(x)\right)$ 在 $\overline{\boldsymbol{F}}_{2}$ 内的一个根, 则条件(6)等价于 $h_{i}(\pi) \neq 0$. 据(5)式有

$$
h_{i}(\pi)=\left\{\begin{array}{l}
h_{1}(\pi), \text { 如果 } i=1 ; \\
h_{2}(\pi)=h_{1}(\pi)+h_{1}^{2}(\pi), \text { 如果 } i \geqslant 2 .
\end{array}\right.
$$

于是得到引理 1 的另一形式如下:

引理 $3 f(x)$ 在 $\boldsymbol{Z} /\left(2^{d}\right)$ 上本原当且仅当

$$
h_{1}(\pi) \neq \begin{cases}0, & \text { 如果 } d=2 ; \\ 0,1, & \text { 如果 } d \geqslant 3 .\end{cases}
$$

下面计算 $h_{1}(\pi)$. 令 $\alpha=\left(\alpha_{t}\right)_{t>0}$ 是 $\boldsymbol{F}_{2}$ 上由 $f_{0}(x)$ 生成的本原序列, 其迹表示为 $\alpha_{t}=$ $\operatorname{tr}\left(\pi^{t}\right)=\sum_{n=0}^{n-1} \pi^{2^{u t}}$, 则在 $G F\left(2^{*}\right)$ 中有

$$
\begin{aligned}
\alpha_{t+i} \alpha_{t+i} & =\sum_{0<u<n-1} \pi^{\left(t+i 2^{u}\right.} \sum_{0<v<n-1} \pi^{\left(t+i k^{\nu}\right.} \\
& =\sum_{0<u<n-1} \pi^{(i+i) 2^{u}} \pi^{u+1 t}+\sum_{0<\omega<v<*-1}\left(\pi^{i 2^{u}+i 2^{\nu}}+\pi^{j 2^{u}+i 2^{\nu}}\right) \pi^{\left(2^{u}+2^{\nu}\right) t} .
\end{aligned}
$$

记 $S$ 为(1)式中确定的指标集, “ $\sum_{(i, i)}$ ”表示关于所有 $i, i \in S, i<i$ 的指标对 $(i, i)$ 求 和, 则

$$
\begin{aligned}
& \sum_{(i, j)} \alpha_{t+i} \alpha_{t+i}=\sum_{0<u<n-1}\left(\sum_{(i, j)} \pi^{(i+j) 2^{u} \pi^{2 u+1}}\right)+\sum_{0<u<v<n-1} \sum_{(i, j)}\left(\pi^{i 2^{u}+i 2^{\nu}}+\pi^{\left.j 2^{u}+i 2^{\nu}\right)}\right) \pi^{\left(2^{u}+2^{\nu}\right)} \\
& =\operatorname{tr}\left(\left(\sum_{(i, i)} \pi^{i+i}\right)^{1 / 2} \pi^{b}\right)+\sum_{0<\omega<v<-1}\left(\sum_{i, j \in S, i \neq i} \pi^{i 2^{u}+i 2^{\nu}}\right) \pi^{\left(2 \omega^{\omega}+2^{\nu}\right) t} \\
& =\operatorname{tr}\left(\left(\sum_{(i, i)} \pi^{i+j}\right)^{1 / 2} \pi^{t}\right)+\sum_{0<m<v<n-1} f_{0}\left(\pi^{2^{u}+2^{v}}\right) \pi^{\left(2^{u}+2^{\nu}\right) t} \text {. }
\end{aligned}
$$

记上式右端为 $\beta_{t}, L$ 为序列左移算于, 视 $\alpha$ 和 $\beta-\left(\beta_{t}\right)_{t>0}$ 为整数序列, 则

$$
f_{\theta}(L) \alpha \equiv 2 \sum_{\langle i, i\rangle}\left(L^{i} \alpha\right)\left(L^{i} \alpha\right) \equiv 2 \beta,(\bmod 4) .
$$

定理 $1 \quad h_{1}(\pi)=\left(\pi f_{0}^{\prime}(\pi)\right)^{-1}\left\{f_{1}(\pi)+\left(\sum_{(i, i)} \pi^{i+i}\right)^{1 / 2}\right\}$.

证据(3)和(8)式有

$$
0=\left(L^{T}-1\right) \alpha \equiv 2 k(L) \beta+2 h(L) \alpha,(\bmod 4),
$$

于是在 $\boldsymbol{F}_{2}$ 上有 $h(L) \alpha=k(L) \beta$. 注意到对 $0 \leqslant u<v \leqslant n-1, k\left(\pi^{2^{\alpha}+2^{v}}\right)-0$, 所以

$$
k(L) \beta=\left\{\operatorname{tr}\left(k(\pi)\left(\sum_{(i, i)} \pi^{i+i}\right)^{1 / 2} \pi^{t}\right)\right\}_{i>0},
$$




$$
h(\pi)=k(\pi)\left(\sum_{(i, i)} \pi^{i+i}\right)^{1 / 2}
$$

最后, 对方程 (2)作形式微商得

$$
x^{T-1}=k^{\prime}(x) f_{0}(x)+k(x) f_{0}^{\prime}(\dot{x}),
$$

从而 $k(\pi)-\left(\pi f_{0}^{\prime}(\pi)\right)^{-1}$, 并且

$$
h_{1}(\pi)-k(\pi) f_{1}(\pi)+h(\pi)-\left(\pi f_{0}^{\prime}(\pi)\right)^{-1}\left(f_{1}(\pi)+\left(\sum_{(i, j)} \pi^{i+i}\right)^{1 / 2}\right) .
$$

至此, 我们得到本文的主要结果:

定理 2 首一多项式 $f(x)-\sum_{i=0}^{c-1} 2^{i} f_{i}(x) \in Z[x]$ 在 $Z /\left(2^{a}\right)$ 上本原当且仅当 $f_{0}(x)$ 在 $\boldsymbol{F}_{2}$ 上本原, 且

$$
\Delta(f) \triangleq\left(\pi f_{0}^{\prime}(\pi)\right)^{-1}\left\{f_{1}(\pi)+\left(\sum_{i, j)} \pi^{i+i}\right)^{1 / 2}\right\} \neq \begin{cases}0, & \text { 如果 } d-2 ， \\ 0,1, & \text { 如果 } d \geqslant 3 ，\end{cases}
$$

其中 $\pi$ 是 $f_{0}(x)$ 在 $\overrightarrow{\boldsymbol{F}}_{2}$ 内的一个根.

推论 $f(x)$ 的本原性由 $f_{0}, f_{1}$ 完全确定. $Z /\left(2^{d}\right)$ 上 $n$ 次本原式的个数为

$$
N(n, d)=\left\{\begin{array}{l}
\varphi\left(2^{*}-1\right)\left(2^{*}-1\right) / n, \text { 如果 } d-2 ; \\
\varphi\left(2^{*}-1\right)\left(2^{*}-2\right) 2^{(d-2) * / n,} \text { 如果 } d \geqslant 3,
\end{array}\right.
$$

其中 $\varphi(\bullet)$ 是 Euler 函数.

注 1 定理 1 中给出的判别式很容易计算.

注 2 引理 3 以前的讨论也对 $Z /\left(p^{d}\right)$ 成立, $p$ 为任意素数.

\section{参考文嗝}

[1] Lidl, R. and Niedereiter, H., Finite Fields, Encyclopedia of Mathematics and lts Applications, Addison-Wesley Publishing Co., 1983.

[2]万哲, 代数和编码, 科学纳版社, 北京, 1976 .

[3] Ward. M., Trans. Amer. Math. Soc., 33 (1931), 153-165.

[4] Ward, M., Trens. Amer. Math. Soc., 35 (1933), 600-628. 